\title{
1 Gestural musical performance with physiological sensors, focusing on the electromyogram
}

Atau Tanaka, Miguel Ortiz

\section{Introduction}

5 Physiological computing has gained currency in recent years as the democratization of 6 biomedical measurement technologies has facilitated take up in interactive computing 7 systems. Signals from the human body can be detected by a wide array of sensors and 8 digitized, providing computational systems information on individual identification, body states, and gross and fine limb movement. These signals have rich potential to be exploited for musical interaction - be it recording performer or audience state in ambient interaction, or capturing instrumentalists' volitional acts in gestural musical interaction. This chapter will

12 focus on the potential of physiological interfaces to capture performer gesture to create embodied interaction with interactive music systems. The chapter is structured as follows: we begin with a brief history of the use of physiological signals in musical performance, noting the evolution of technology that has enabled the current interest. We introduce the range of physiological signals, and focus on one, the electromyogram, that reports muscle tension. We present techniques for using the EMG in music, including signal pre-processing in the form of feature extraction, and analysis in the form of machine learning. We discuss challenges of reproducibility and situate the EMG in multimodal context with other sensing modalities. We conclude by proposing gesture "power" as one low level feature that in part represents Laban's notion of "effort" to demonstrate the potential of the EMG to capture expressive musical gesture.

Human brainwaves were first measured by Berger in 1924, then sonified in the 1930s by physiologists Adrian and Mathews who translated signals measured with electrodes into audio signals to be heard through loudspeakers. In 1965, the American composer Alvin Lucier used brainwaves to activate acoustic percussion instruments in Music for Solo Performer. This work with biosignals was adopted by composers such as Richard

28 Teitelbaum and David Rosenboom (Brouse et al., 2005), leading to the publication of the seminal book Biosignals and the Arts: Results of Early Experiments (Rosenboom, 1976). John Cage, in his large scale work, Variations 7, performed in 1966 in New York City as part 
31 of the Experiments in Art \& Technology (E.A.T.) 9 Evenings, called for "body sounds: heart, 32 brain, lungs, stomach." In 1974, Manford Eaton published Bio-Music, a manifesto calling for 33 a new biofeedback-based art in which the intentions of the composer are 'fed directly' to the

34 listener (Eaton, 1974).

35 With the arrival of early digital systems in the early 1990's, musicians began to interface 36 digitized biosignals with MIDI controlled synthesizers and computer music systems. The 37 IBVA $^{1}$ was created by Masahiro Kahata and was an early low-cost, portable digital EEG 38 device for art. The BodySynth ${ }^{2}$, created by Chris Van Raalte and Ed Severinghaus was an 39 EMG music interface that was used performance artists such as Pamela $\mathrm{Z}$ and Laurie 40 Anderson. Ben Knapp and Hugh Lusted, researchers at Stanford University's CCRMA 41 created the BioMuse ${ }^{3}$ in 1990, a DSP based biosignal-MIDI interface that read EEG, EOG, 42 and EMG (Knapp \& Lusted, 1988). This system, and its subsequent minituarized incarnations 43 were adopted by the two authors for a series of music concert pieces. This facilitated 44 replication and rapid prototyping of systems that has facilitated ensembles such as the 45 BioMuse Trio, and use of biosignals in groups to conduct audience studies (Ortiz, 2012). The increasing practicality of electronics resulted in use in media art contexts by Nagashima

47 (Nagashima, 2003). The information sharing in DIY communities has led to the publication 48 of designs for mechanomyogram (MMG) pickups ${ }^{4}$ that have been used as the basis for 49 Donnarumma's Xth Sense interface. $^{5}$

Advances in materials, electronics miniaturization, and manufacturing, have resulted in a spate of consumer products that interface the human body to computing systems. These products range from forms of biofeedback to aid in personal health monitoring ${ }^{6}$, to products that exploit insight from research in affective computing ${ }^{7}$. Sophisticated multichannel, multimodal devices have been brought to market for hobbyists to control model drones by gesture, and mundane tasks such as a virtual slide presentation clicker ${ }^{8}$.

\footnotetext{
${ }^{1} \mathrm{http}: / /$ www.ibva.co.uk

${ }^{2} \mathrm{http}: / /$ www.synthzone.com/bsynth.html

${ }^{3} \mathrm{http}: / / \mathrm{www}$.biocontrol.com

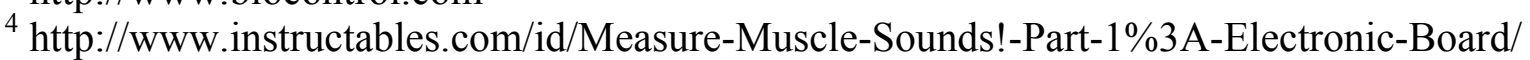

${ }^{5} \mathrm{http}: / /$ www.xth.io/

${ }^{6} \mathrm{http}: / / \mathrm{www} \cdot$ mindmedia.info/

${ }^{7} \mathrm{http}: / /$ www.empatica.com/

${ }^{8} \mathrm{http}: / /$ www.myo.com/
} 
56 As the technologies became more accessible and accurate, the artistic focus of these projects has shifted from an initial focus on biofeedback, to the notion of bio-control, to direct physiological interfacing. Biosignals have broad musical potential. They range from their use in augmenting traditional musical instrument performance, to copying existing instruments for virtual, "air" instruments, to the creation of entirely new instruments in areas of contemporary music and NIME. Free space gesture can be used to track non-instrumental gesture, such as dancing and conducting (Marrin \& Picard, 1998). All these types of musical interaction can find application in music pedagogy and movement rehabilitation medicine.

\section{Biosignals}

65 The term biosignals is used to refer to physiological signals from living organisms, and that manifest change in electrical potential across a specific cells, tissues, or organs. Tissues, such as nerves, skeletal muscles, cardiac muscle and soft muscles, can generate electrical activity, whereas others, like the skin and the eyes, are electrically passive and manifest potential differences. The change in electrical potential offers a direct correlate of physiological activity and depends on the entity being monitored. The most common biosignals are: Galvanic Skin Response (GSR), which is the change of the skin's electrical conductance properties caused by changes in emotional states like stress; Electrocardiogram (ECG/EKG), a measurement of the electrical activity of the heart; Electrooculogram (EOG) is the measurement of the Corneal-Retinal Potentials (CRP) across the eye; the Electroencephalogram (EEG) monitors the electrical activity caused by the firing of cortical neurons across the brain's surface; and the Electromyogram (EMG) which is the electrical signal generated from activation and contraction of skeletal muscle fibers.

\subsection{The electromyogram}

Electromyography represents the isotonic, isometric and isokinetic muscle activity generated by motor neurons (De Luca \& Van Dyk, 1975). Isometric activity is muscle tension with no change in muscle length (such as holding up weights without movement). Isotonic activity is that where the change of muscle length and resistance is constant (push ups, curling dumb

83 bells). Isokinetic activity is that where the speed of movement and total tension are constant but tension and length of muscle might change (fast pull-ups). 
85 Motor Unit Action Potential (MUAP) refers to the activation of motor units. Motor units are defined as the combination of a motor neuron and the muscle fibers it innervates. The MUAP therefore is the electrical firing of a motor unit. This impulse is then transmitted from the motor neuron to the muscle causing it to contract. The repetitive firing of a motor unit creates an impulse train, which allows for what we perceive as sustained muscular contraction.

90 The main mechanisms that control the magnitude and density of the measured signal are the recruitment and firing rate of MUAPs within the measured muscle in the area under study. Individual MUAPs' have an average amplitude of $100 \mu \mathrm{V}$. The EMG signal that results from the firing of individual MUAPs is stochastic in nature, reflecting the fact that the motor units recruited in any gestures is constantly changing and has an amplitude range of $+/-5 \mathrm{mV}$ before further amplification. Typical frequency contents of raw EMG signals for healthy individuals ranges between 0 and $500 \mathrm{~Hz}$, with dominant energy concentration in the 50-150 $\mathrm{Hz}$ range.

\subsection{Signal acquisition}

EMG signals are measured using electrodes which are conductive probes placed on the body. When a biopotential is created by the contraction of the muscle, a differential amplifier picks up the voltage changes between the otherwise equipotential areas created by the electrodes. There are two main types of electrodes, invasive and non-invasive. Invasive electrodes are needles or thin wires that measure activity of deep muscle fibers to be able to report the activity of individual MUAPS. The placement of the electrodes is time consuming, must be carried out by healthcare professionals. This, compounded by the relative discomfort wearing them make them ill suited for musical performance practice.

107 Non-invasive electrodes make electrical contact with muscle cells through the surface of the

108 skin, providing surface EMG (sEMG). The most common electrodes are silver/silver chloride 109 wet gel electrodes. These types of electrodes have the best skin impedance values and are the 110 standard in medical practice. Altough they have been used extensively in music performance, 111 they require preparation of the skin and are prone to noise associated with movement 112 artefacts from the snap fasteners.

113 Dry electrodes have become increasingly common in high end as well as low-cost consumer 114 systems. The electrical contact with the skin via gold plated or other highly conductive metals 115 approaches, but does not equal that of gel electrodes. However, most designs incorporate 
116 active pre-amplifiers immediately adjacent to the electrodes, making the output more robust

117 to electromagnetic interference and preventing the accumulation of noise along the signal

118 cable. This plus the ease of application and re-application make dry electrodes practical for

119 the use of biosignals in interactive arts practice.

120 Electrodes are typically placed at the middle portion of the studied muscle belly (Hermens,

121 Freriks, Disselhorst-Klug, \& Rau, 2000). The most common placement of EMG sensors for

122 musical practice is in the forearms of the performer. This is a convenient place for the sensors

123 as it allows finger activity to be tracked via the forearm flexor and brachioradialis muscles

124 without an intrusive sensors, such as gloves, on the hands. Action potentials measured by

125 electrode pairs are processed using the differential amplification measuring voltage at the two

126 sites, subtracting the signals and amplifing the resulting difference. Any signal component

127 that is present at both electrodes is removed through common mode rejection. When using

128 wet electrodes, it is common practice to use an electrode placed in an unrelated area to act as

129 a reference ground, such as the ear lobe, or joints and other bony areas where no action

130 potentials are likely to occur.

131 Ambient noise such as that originating in mains lines and power sources can be problematic

132 when measuring biosignals. The alternating current (AC) frequency of mains electricity is

133 centered around $60 \mathrm{~Hz}$ (Americas) or $50 \mathrm{~Hz}$ (Europe). A notch filter can be used to attenuate

$13450 / 60 \mathrm{~Hz}$ noise but good filter design is necessary since this overlaps with dominant energy

135 frequency of meaningful EMG, $50-150 \mathrm{HZ}$. The body itself can be a source of artifacts - the

136 heart is a strong muscle with a dominating EMG signal. This can interfere with EMG

137 measurements from limbs if the differential electrical measurement takes place across the 138 chest area.

\section{$1392.3 \quad$ Signal processing and feature extraction}

140 The EMG signal has been compared in its richness to audio, making audio signal processing

141 and pattern recognition techniques potentially relevant in analyzing the biosignal. The EMG

142 is, however, ultimately not a continuous signal, but the sum of discrete neuron impulses. This

143 results in an aperiodic, stochastic signal that poses challenges to audio-based signal and

144 information processing. For interactive music applications to track performer state or gesture,

145 some kind of signal analysis needs to take place. This could typically be in two stages, first

146 feature extraction, followed by classification or mapping. 
147 Feature extraction of the EMG entails analyzing the raw signal and using signal processing to

148 create a representation containing salient higher level information about the nature of the

149 gestural content. In this light, and given its signal complexity approaching that of audio, it

150 can make use of techniques not dissimilar to those used in audio signal processing for music

151 information retrieval. However, given the aperiodic stochastic nature of the EMG signal and

152 the distinct nature of gestural content compared to musical content, the actual relevant signal

153 processing techniques and high level information gleaned, will differ.

154 EMG signal features can be grouped into time domain (TD) and frequency domain (FD).

155 Given the aperiodic nature of the EMG, there is no harmonic content for FD algorithms to 156 extract, making them less useful for EMG analysis. The most straightforward TD feature is 157 amplitude estimation, which reflects the level of muscle tension. This can be achieved in a 158 number of techniques of differing sophistication. Simple amplitude estimation can be 159 achieved as envelope following by smoothing, or low pass filtering of the raw EMG data.

160 This can be done, for example, by taking the median of the signal over a time window, but 161 introduces latency and lag of the length of the median window. Root Mean Square (RMS), 162 the square root of the mean of the squares of samples in a time series, is a calculation of 163 electrical power, and relates to constant force and non-fatiguing muscle contraction.

164 Recursive Bayesian estimation or Bayes filter, is a probabilistic approach for estimating an 165 unknown function over time using incoming measurements. The algorithm has been applied 166 as a nonlinear estimator of EMG amplitude that improves the signal-to-noise ratio compared 167 to RMS and considerably stabilizes the signal while remaining reactive to fast transients.

168 Other TD features that show good performance are Mean Absolute Value which provides 169 energy information of the signal; Waveform Length which provides the cumulative length of 170 the waveform over the time segment, and is related to the complexity of the EMG signal and 171 whose values indicate a measure of waveform amplitude, frequency and duration all within a 172 single parameter; and Willison Amplitude as a measure of frequency information of the 173 signal, similar in nature to the number of zero crossings and related to the firing of MUAPs 174 and muscle contraction force.

175 Frequency Domain features are based on statistical properties of the EMG signal's power 176 spectrum density. FD features are usually used to detect neural abnormalities and muscle 177 fatigue as it is related to motor unit recruitment. The most common FD features are: Median 178 Frequency which is the frequency at which the spectrum is divided into two regions with 
equal amplitude; Peak Frequency which is the frequency at which the maximum power

180 occurs; and the Mean Frequency, also known as Spectral Centroid, the center of gravity line

181 of the spectrum. FD features are useful for monitoring muscle fatigue as it results in a

182 downward shift of the spectrum as fatigue increases. It has been noted that FD features

183 perform poorly when compared to TD features for gesture classification tasks (Phinyomark et

184 al., 2013).

\section{Musical mapping}

186 Musical transformation of the EMG can take place at a number of different levels. The

187 physiological signal can be used directly as musical material in processes of sonification. The

188 EMG can be used in a control paradigm by mapping post-processed biosignal input directly

189 to sound synthesis parameters. The EMG data can be transformed into other discrete or

190 continuous presentations through machine learning.

\subsection{Sonification}

192 A common sonification technique is audification, where the raw data stream is upsampled

193 and fed directly to speakers or further processed by audio DSP modules. This is a direct way

194 of 'listening' to the signal itself which is characterized as a very low frequency sound with

195 noisy timbre. Once the signal is in the audio domain, it can be treated and transformed as any

196 other sound source.

197 The procedure is prone to artefacts. EMG hardware systems typically sample at a frequency

198 between $100 \mathrm{~Hz}$ and $2 \mathrm{kHz}$. When upsampling to audio sampling rates (44.1 or $48 \mathrm{kHz}$ ), this

199 will generate extra samples not present in the original signal, resulting in a sample-and-hold

200 effect where after a value will be repeated in the sound signal until a new EMG value arrives.

201 This translates into spurious high frequency content. A low pass filter at $200 \mathrm{~Hz}$ is useful in 202 avoiding this problem.

\section{$203 \quad 3.2 \quad$ Mapping}

204 The raw EMG signal can be processed or subjected to feature extraction to provide lower

205 bandwidth data for discrete musical event and continuous sound synthesis control. Event

206 triggers can be derived from the rectified EMG amplitude. The high frequency, rapidly

207 varying nature of the signal, however, make it prone to false or multiple triggering. Using 
smoothing techniques to filter transient spikes may introduce latency, causing system responsiveness to suffer. A practical solution is to use a Schmitt trigger implementation, where a hysteresis loop prevents an upward going event threshold to re-trigger until a lower, downward going threshold has been attained.

The EMG is well suited for the continuous control of sound synthesis parameters. Multiple channels can be used in conjunction with one another to generate series of events whose sustaining sounds were shaped by subsequent muscle gesture. Gesture-parameter mapping strategies, such as "one-to-many" mapping, where a single sensor input it mapped to multiple synthesis parameters, or "many-to-one" where multiple sensor inputs might be combined to control a single synthesis parameter (Hunt \& Wanderley, 2003), are highly relevant to using EMG in sound synthesis control. The amplitude feature, described above, is most commonly used, with different low pass, or Bayesian filters to fine tune the smoothness and responsiveness of the system. Calibration, by setting a minimum and maximum range of the signal to utilize aids in eliminating a noise floor and allows focusing on a specific amplitude range. Different response curves, from linear, to logarithmic, to exponential, can modify the input/output relationship, creating a more natural feeling mapping between the exertion of the performer and the facility of musical change.

The use of multiple EMG channels in a ring formation around the upper forearm is common in myoelectric control systems and interactive performance applications. Each channel provides information related to muscle activation in the area of each electrode pair. This approach allows for the activation of opposing muscle groups to be monitored independently and creates a more expressive feature space. Wrist flexion and extension can be effectively tracked with just two channels of EMG on opposing forearm muscle groups. Four or more channels around the forearm can provide information on hand rotation. Furthermore, it makes the possibility to analyze the relationships in activation for each signal which can give more detailed information of individual finger movements and hand gestures (Saponas et al., 2009).

\subsection{Machine learning}

Beyond direct parameter mapping, the complexity of the EMG signal points to the potential for using pattern recognition methods and information analysis techniques to both gain lower level understanding of limb and sub-limb movement and elicit higher order representations of 
musical gesture. These representations can be continuous in nature, or discrete in the recognition performed.

241 Regression is one way for data analysis to produce continuous interaction by modeling the relationship between a scalar dependent variable and one or more explanatory variables. It transforms one continuous data stream into another information stream. Regression is useful

244 for dimensionality reduction of the EMG, either taking multiple streams of raw data to

245 produce information in fewer and more pertinent parameter dimensions for sound synthesis.

246 Discrete labels can be assigned to blocks of continuous EMG data, identifying gesture.

247 Machine learning classification techniques have been applied to EMG signals in biomedical research for rehabilitation and basic prosthetics control applications. The requirements of these scenarios are quite distinct from musical applications. Most of these applications use classifiers like support vector machines (SVM) to distinguish between different hand positions. The task is a supervised learning task where the classifier is trained with a series of examples for subsequent linear, binary clustering. Ultimately, classification of static positions can be thought of not as gesture recognition, but posture classification, where a static steady state EMG signal from a number of EMG channels giving signatures of different hand positions. The fact that these static positions, and therefore the classification result, are achieved at the end of a gesture mean that in musical applications, this would be experienced as a sort of latency, where the classifier reports on the gesture after it is completed.

The success criteria of gesture recognition in these different application areas is driven by characteristics essential to the application. In prosthetics control, reducing error is primordial, resulting in what might seem like relatively simplistic, binary controls. In music on the other hand, the dynamic time based nature of musical performance, where musical expression arises from subtle variation of gesture, generates a completely different set of needs in terms of latency, continuity, and invariance. In music, a gesture needs to be recognized before it is finished - a nontrivial challenge. We need to recognize without suppressing variation, completely inverting the suppression of variation in canonical examples of data classification such as handwriting or speech recognition. And finally, we seek forms of continuous interaction with musical output such as sound synthesis, rather than discrete event triggering. For the first challenge, techniques of "early recognition" can be implemented. For the second, new adaptation approaches may be relevant, and for the third, regression techniques are useful. 
We created an early EMG gesture classifier using the K-Nearest Neighbors algorithm to train and subsequently distinguish in real time six different hand gestures using two channels of

273 forearm EMG (A Tanaka \& Fistre, 2008). Currently, we are applying recent advances in

274 machine learning to the EMG and other physiological signals. The use of Particle Filtering

275 techniques allows continuous reporting of recognition probability against a training set and

276 real time tracking of gestural variation against the reference template (B. Caramiaux,

277 Montecchio, Tanaka, \& Bevilacqua, 2014). The temporal sensitivity, however, require

278 segmentation or some external signal indicating gesture onset.

\section{Musical gesture}

280 The complexity of EMG data poses specific challenges and presents opportunities in live 281 interactive performance applications. A single data stream can embody information about 282 multiple parts of the body. A muscle group controls the movement of limbs below it: one 283 signal from forearm muscles can contain information multiplexing the movement of five 284 fingers. Meanwhile the EMG signal may not return to a rest state in between a sequence of gestures. One gesture may begin before the previous one ends. The EMG signal, therefore, may encapsulate information on multiple parallel, complex gesture, as well as a sequence of serial, compound gesture. This creates the need for techniques of gesture segmentation and decomposition. In addition to distinguishing constituent gesture primitives within compound and complex gestures, fundamental challenges exist to differentiate between voluntary, involuntary, and incidental physical behavior. These qualities have a bearing on musical applications, the kind of gesture recognition techniques and sound synthesis mappings that can be applied.

293 The feature extraction and data analysis techniques described above allow the music

294 interaction researcher to process the richness of the EMG signal to make it more usable.

295 However, because of the number of different muscle groups solicited for making any action, 296 there does not exist a direct correspondence between muscle exertion and resulting limb 297 movement. This means that there is not a direct, deterministic relationship between muscle states and gesture, making movement representation uniquely via EMG difficult. Two aspects contribute to this problem: the fact that multiple different combinations of muscle tension could result in the same gesture, and the fact that muscle tension may not result in movement at all. 


\subsection{Gesture without movement}

303 The fact that EMG measures isotonic and isometric activity means that there is not a

304 deterministic link between limb movement and muscle tension. Muscle tension may be exerted without corresponding limb movement. Conversely, certain gross gestures could take place with little muscle contraction. While this makes prediction of movement gesture difficult, these characteristics of the EMG signal make it uniquely suited for detecting gesture preparation. This makes the EMG an interesting sensing modality that offers complementary information to physical sensors that report on movement after it is performed. In this sense, the EMG is useful in contexts of multimodal interaction.

\subsection{Redundancy and ambivalence}

312 While the EMG offers these unique opportunities, these qualities can make it challenging to

313 implement in scenarios or studies with non-specialist users. The "same" (or highly similar)

314 gross movement can be made by invoking any number of muscle tension trajectories. This

315 can be thought of like the multiple degrees of freedom problem found in robotics in multi-

316 joint articulation and is formalized by the neurophysiologist Nikolai Bernstein in motor

317 control theory. This poses a distinct problem in applying the machine learning techniques

318 described above. Different people will invoke different combinations of muscles to perform

319 what to an observer is the same gesture. Complicating matters further, a single subject may

320 perform the same movement with slightly different muscle tension profiles each time. This

321 poses the problem of gesture reproducibility. Additionally, repeated performance of a given

322 set of gestures will lead to muscle fatigue which adds further aperiodic variations to the

323 measured signal. While FD features can be used to monitor the evolution of fatigue over

324 time, the effects of fatigue in the signal's amplitude are not directly correlated.

325 We have conducted experiments that study the ability of lay users to learn and copy gestures

326 (Baptiste Caramiaux, Donnarumma, \& Tanaka, 2015). We designed gesture vocabularies that

327 focused on different muscle groups in simple lower arm and wrist movement tasks. We

328 provided auditory feedback that allowed the subject to monitor their gesture during

329 execution. Later, in separate work with more complex gestures, we have observed that

330 visualization of multiple channels of EMG signal aid the subject in replicating a gesture with

331 sufficient muscular similarity to be successfully classified by machine learning techniques. 


\subsection{Multimodality}

333 The ability of EMG to report on gesture without movement, alongside the degrees of freedom 334 problem, mean that the EMG is well suited for use in context with other sensing modalities.

335 Multimodal interaction in HCI takes advantage of the integration of different input modalities 336 to enhance the information bandwidth with an interactive system. The EMG, reporting on the 337 intention and preparation of a gesture provides a complementary input modality to inertial 338 sensors or motion capture systems that report on the physical movement result of that gesture.

339 We have used muscle sensing alongside optical motion capture and accelerometer sensing to

340 look at this sensor complementarity in the execution of musical gesture (Donnarumma,

341 Caramiaux, \& Tanaka, 2013). We observed several different relationships between the 342 sensing modalities: synchronicity when certain segments of the sensor data are coincident 343 across modalities; coupling, where different modalities vary in similar ways in different 344 articulations of the same gesture; and correlation, where the actual relationship between 345 different sensing modalities shifts during the course of the gesture.

\section{Expressivity}

347 These relationships of multimodal interaction put EMG in context for providing an 348 expressive dimension to gestural interaction in music. The complementary sensor modalities could be used in an orthogonal fashion, where the same muscle tension trajectory could mean different interactions on the music based on different gross positions detected by another sensing modality. We have explored techniques for multi-modal interaction to distinguish similar muscular gestures in different points in space_(Atau Tanaka \& Knapp, 2016).

Biosignals can also be seen as extremely low latency information about gesture. The EMG reports a neural command that causes muscle tension, resulting in limb movement. It reflects the intention of gesture, and in this regard is at the opposite end of movement production than a sensor such as an accelerometer that reports on physical artifacts resulting from gesture. A classical sensor, then, is at the "output" of a gesture while the EMG is a signal that is the "input" to a gesture.

359 The execution of gesture can take place in free space or with "boundary objects". These

360 boundaries could be objects one grasps, like a physical implement or instrument, or a surface that offers resistance to movement. Boundary objects therefore offer isotonic gestures resistance against which they may act, facilitating the adjustment of exertion. This provides a 
363 form of feedback that facilitated task performance in our study (Baptiste Caramiaux et al.,

364 2015). Through this study, we identified the abstract notion of gesture "power" which could 365 be tracked by the EMG and detected in certain signal features of the EMG. On surface

366 gestures, the notion of power corresponds to exertion, or effort. This creates interesting links

367 with the concept of "effort" in Laban Movement Analysis movement (Silang Maranan et al., 368 2014).

369 The characteristics of the electromyogram signal make it uniquely well suited for capturing expressive musical gesture. The challenges it poses - the degrees of freedom problem, and the lack of direct link between signal and resulting movement - provide the richness in the signal that cannot be gleaned from other, physical sensors. The signal is at the very source of gestural activation, making it now just responsive, but able to report on musical intention. It is ultimately not an external observation of the physical output of musical movement, but the body's own signal for creating musical gesture.

\section{References}

377 Brouse, B., Castet, A., Filatriau, J., Lehembre, J. J., Noirhomme, R., \& Simon, Q. (2005). $378 \quad$ From Biological Signals to Music.

379 Caramiaux, B., Donnarumma, M., \& Tanaka, A. (2015). Understanding Gesture Expressivity through Muscle Sensing. ACM Transactions on Computer-Human Interaction, 21(6), 126.

Caramiaux, B., Montecchio, N., Tanaka, A., \& Bevilacqua, F. (2014). Adaptive Gesture Recognition with Variations Estimation for Interactive Systems. Transactions on Interactive Intelligent Systems, 4(4).

De Luca, C. J., \& Van Dyk, E. J. (1975). Derivation of some parameters of myoelectric signals recorded during sustained constant force isometric contractions. Biophysical Journal, 15(12), 1167-1180.

Donnarumma, M., Caramiaux, B., \& Tanaka, A. (2013). Body and Space: Combining Modalities for Musical Expression. Conference on Tangible and Embedded Interaction (TEI), Work-in-progress.

Eaton, M. L. (1974). Bio-music. K: Something Else Press. recommendations for SEMG sensors and sensor placement procedures. Journal of 
395

Hunt, A., \& Wanderley, M. M. (2003). Mapping performer parameters to synthesis engines. Organised Sound, 7(02), 97-108.

Knapp, R. B., \& Lusted, H. S. (1988). Biomuse: Musical performance generated by human bioelectric signals. The Journal of the Acoustical Society of America, 84(S1), S179.

Marrin, T., \& Picard, R. (1998). The "Conductors Jacket": A Device for Recording Expressive Musical Gestures. In Proceedings of the International Computer Music Conference (pp. 215-219).

Nagashima, Y. (2003). Bio-sensing systems and bio-feedback systems for interactive media arts. In NIME'03 (pp. 48-53). National University of Singapore Singapore, Singapore.

Ortiz, M. (2012). A Brief History Biosignal-Driven Art: From biofeedback to biophysical performance. eContact!, 14(2).

Phinyomark, A., Quaine, F., Charbonnier, S., Serviere, C., Tarpin-Bernard, F., \& Laurillau, Y. (2013). EMG feature evaluation for improving myoelectric pattern recognition robustness. Expert Systems with Applications, 40(12), 4832-4840.

Rosenboom, D. (1976). Biofeedback and the Arts, Results of Early Experiments. Vancouver, BC, Canada: Aesthetic Research Centre of Canada, A.R.C.

Saponas, T. S., Tan, D. S., Morris, D., Balakrishnan, R., Turner, J., \& Landay, J. A. (2009). Enabling always-available input with muscle-computer interfaces. In Proceedings of the 22nd annual ACM symposium on User interface software and technology (pp. 167-176). Silang Maranan, D., Fdili Alaoui, S., Schiphorst, T., Pasquier, P., Subyen, P., \& Bartram, L. (2014). Designing for Movement: Evaluating Computational Models Using LMA Effort Qualities. Proceedings of the 32Nd Annual ACM Conference on Human Factors in Computing Systems, 991-1000.

Tanaka, A., \& Fistre, J. (2008). Method and apparatus for analysing gestures produced in free space, e.g. for commanding apparatus by gesture recognition. Unites States Patent.

Tanaka, A., \& Knapp, R. B. (2016). Multimodal Interaction in Music Using the Electromyogram and Relative Position Sensing. In M. Lyons \& A. R. Jensenius (Eds.), A NIME Reader. Springer. 